\title{
La comunicación para el desarrollo y sus nuevos desafíos
}

\section{Resumen}

Muchos de los temas centrales de la agenda del desarrollo y de la comunicación para el desarrollo siguen siendo los mismos, pero, ciertamente, han aparecido nuevos escenarios, nuevas relaciones de cooperación, nuevos temas y nuevos actores que han reconfigurado el mundo en este nuevo siglo y han colocado nuevas preocupaciones y desafíos. Para enfrentar estos retos, es importante impulsar la construcción de una cultura para el desarrollo que se sostenga sobre la base de una democracia inclusiva, con ciudadanía activa y que garantice gobernabilidad.

Palabras clave: Comunicación para el desarrollo, Comunicación para el cambio de comportamientos, Comunicación para el cambio social, Cultura para el desarrollo, Democracia inclusiva.

\section{Communication for development and its new challenges Abstract}

Most main issues on the Development and Communication for Development Agenda remain the same, but new scenarios, new cooperation partnerships, new issues and new players have arisen thus reshaping the world in this new century and posing new concerns and challenges. In order to face such challenges, promoting building a culture for development to be sustained on the basis of an inclusive democracy with active citizenship guaranteeing governance is important.

Key words: Communication for development, communication for behavior change, communication for social change, culture for development, inclusive democracy

Segundo Armas Castañeda

Recibido el 17 de septiembre de 2014

Aceptado el 30 de octubre de 2014 
El largo debate que ha rondado el ambiente intelectual y el espacio de las prácticas comunicativas latinoamericanas en el campo del desarrollo podría resumirse en dos perspectivas concretas: comunicación para el cambio de comportamientos y comunicación para el cambio social. El cambio de comportamientos corresponde a una perspectiva funcionalista, pragmática y efectista que pretende alcanzar resultados estadísticos positivos de cambio de conducta en el corto plazo, pero carece de sostenibilidad. El cambio social corresponde a un enfoque de cambios estructurales de mediano y largo plazo, pero como enfoque es denso y poco viable para responder a las urgencias comunicacionales.

Sin embargo, más allá de los análisis y discusiones teóricas, la experiencia concreta en el campo de la comunicación para el desarrollo nos enseña que sí es posible conciliar ambas perspectivas para atender los problemas del desarrollo, en el corto, mediano y largo plazo. Por cierto, esta conclusión no se produce solo desde la reflexión y el discurso teórico o académico acerca de la comunicación para el desarrollo, sino que se origina como consecuencia de las constataciones o vivencias concretas en el campo de los proyectos de desarrollo.

Estamos seguros que cambiando comportamientos podemos avanzar también en la construcción del cambio social. En todo caso, el cambio de comportamientos debe concebirse como el punto de partida de un largo proceso de construcción de cambio de mentalidades que más que cambiar conductas, alude a cambios culturales de carácter estructural.

Esto quiere decir que, en la apuesta del cambio social, el cambio conductual constituye una de las dimensiones necesarias para avanzar en un cambio cultural más profundo que debe cristalizarse, finalmente, en la construcción de una cultura para el desarrollo.

Todas las reflexiones han coincidido unánimemente en considerar que el centro y el fin del desarrollo es la gente, y, allí, el cambio de mentalidades es fundamental para empoderarla como actor o protagonista que moviliza sus voluntades y compromisos que le permita construir el destino que le pertenece y que le es propio.

Allí radica el desafío de la comunicación para el desarrollo. Y aquí es oportuno relevar una importante propuesta de la Conferencia Intergubernamental sobre Políticas Culturales para el Desarrollo, 
realizada en Suecia en 1998, bajo el auspicio de la Organización de las Naciones Unidas para la Educación, la Ciencia y la Cultura (Unesco), en la que los gobiernes participantes acordaron poner más atención al papel de la cultura en los procesos de transformación social ${ }^{1}$. Asimismo, y en este mismo sentido, rescatamos el espíritu de impulsar una cultura para el desarrollo que planteara Javier Pérez de Cuéllar durante su gestión como Secretario General de las Naciones Unidas, entre 1982 y 1991.

La tarea del desarrollo y de la comunicación para el desarrollo tiene al frente varios desafíos que afrontar de cara a los Objetivos de Desarrollo del Milenio (ODM) y a la agenda de desarrollo post-2015. El primer informe sobre la agenda mundial de desarrollo post-2015, denominado "El futuro que queremos para todos", plantea "un enfoque de políticas integradas para garantizar el desarrollo económico y social inclusivo y la sostenibilidad ambiental en una agenda que responda a las aspiraciones de todas las personas de un mundo libre de miedo y necesidad". El informe plantea cinco recomendaciones:

- La visión de desarrollo para el futuro debe estar centrada en los principios de derechos humanos, igualdad y sostenibilidad.La agenda debe estar basada en objetivos y metas concretas orientadas al logro de resultados.

- Para alcanzar el futuro que queremos para todos, va a ser necesario un alto grado de consistencia en las políticas públicas a nivel global, regional, nacional y sub-nacional.

- La agenda de desarrollo post-2015 debe ser concebida como una agenda verdaderamente global con responsabilidades compartidas entre todos los países.

- Es muy pronto para definir metas y objetivos concretos para la agenda de desarrollo post-2015; para esto, varios procesos tienen que correr su curso. Las decisiones que fueron tomadas en la Cumbre sobre el Desarrollo Sostenible, Rio+20, y el seguimiento

${ }^{1}$ Informe de la Conferencia intergubernamental sobre políticas culturales para el desarrollo (1988). Suecia: UNESCO, p. 16. 
a estas decisiones serán una guía importante para la discusión sobre la agenda de desarrollo post-2015, y esta última tendrá que estar cabalmente alineada a esas decisiones.

Muchos de los temas centrales de la agenda del desarrollo y de la comunicación para el desarrollo, como la erradicación de la pobreza, siguen siendo los mismos, pero ciertamente, han aparecido nuevos escenarios, nuevas relaciones de cooperación, nuevos temas y nuevos actores que han reconfigurado el mundo en este nuevo siglo y han colocado nuevas preocupaciones y desafíos. La agenda para el desarrollo post-2015 considera algunos retos emergentes de desarrollo que incluyen la persistencia de grandes desigualdades, la brecha del conocimiento entre países y al interior de los países; rápidos cambios demográficos; una creciente huella ecológica; temas relacionados con la paz y seguridad y déficits de gobernanza a nivel global, nacional y sub-nacional.

Las relaciones de cooperación se dan de manera concertada y se rigen bajo el sentido o principio de la responsabilidad compartida en torno a problemáticas comunes que están enmarcadas en los ODM, y, próximamente estarán presentes también en la agenda para el desarrollo post-2015. Ciertamente, en los últimos años se ha reducido sustancialmente la cooperación no reembolsable debido a la crisis financiera internacional que ha golpeado la economía de los países donantes más importantes del mundo y ha afectado a muchas organizaciones no gubernamentales que han venido canalizando dichos recursos y se han visto obligadas a redefinir sus ejes de acción y sus estrategias de financiamiento.

La cooperación internacional se da ahora como una estrategia concertada entre los países del Norte y los países del Sur para enfrentar juntos los desafíos del mundo globalizado donde aún persisten los problemas de la pobreza y las desigualdades, las pandemias, la migración, la privación de derechos, el cambio climático, la exclusión social, entre otros. Por eso, los nuevos términos de la cooperación internacional tienen que tener un contenido transformador y deben focalizarse en el logro del 
desarrollo humano, generando capacidades y oportunidades, en un clima de libertad.

En este desafío de impulsar una cultura para el desarrollo humano sostenible, nos parece fundamental promover procesos que nos permitan construir una democracia inclusiva, con ciudadanía activa y que garantice gobernabilidad. Otros temas retadores para la comunicación para el desarrollo tienen que ver también con cómo enfrentamos los retos que surgen como consecuencia del calentamiento global y el cambio climático, cómo asumimos una perspectiva de género basado en la equidad y de respeto al "otro", y, cómo manejamos adecuadamente las bondades y los problemas que traen consigo las nuevas tecnologías de información y comunicación.

En este nuevo escenario, la tarea de construir una democracia inclusiva nos remite a reflexionar sobre uno de los modelos de desarrollo económico más aplicados en nuestros países latinoamericanos: el modelo del crecimiento económico.

El modelo de desarrollo basado en el crecimiento económico, pero carente de una política de redistribución del ingreso, ha mostrado serias limitaciones en todos los países latinoamericanos que lo han aplicado y lo vienen aplicando. A pesar que en la década de los noventas, la Comisión Económica para América Latina y el Caribe (Cepal) dio un giro muy importante pasando del modelo de industrialización mediante la sustitución de importaciones al de la transformación productiva con equidad, en un claro tránsito del modelo endógeno hacia el modelo exógeno a la luz de la economía globalizada; los gobiernos de la región han persistido en la apuesta por el crecimiento económico y muy poco se han preocupado porque los beneficios de este crecimiento lleguen a las amplias mayorías de la población donde aún están atrincheradas las columnas más fuertes de la pobreza y extrema pobreza.

Durante la primera vuelta electoral, el candidato Ollanta Humala abanderó el proyecto de la gran transformación que recogía las legítimas aspiraciones de los sectores sociales más olvidados del país, pero, para asegurar su victoria en la segunda vuelta electoral tuvo que ampliar su base de apoyo y sumar a las fuerzas del toledismo que lo obligó a girar 
hacia el centro y enarbolar una hoja de ruta ${ }^{2}$ que desde todo punto de vista constituía, aparentemente, un acuerdo programático nacional básico y una oportunidad sobre la cual podía concretarse un pacto social de gobernabilidad para emprender algunas reformas importantes para el país. La hoja de ruta y el mensaje de un gobierno comprometido con la inclusión social eran señales de que podía marcar la diferencia con los gobiernos anteriores, cuyas agendas políticas tuvieron como prioridad el impulso de la economía de mercado y el crecimiento económico, pero no se preocuparon o lo hicieron muy poco por la redistribución del ingreso y la inclusión social.

Es cierto que en los últimos años la economía del país ha venido creciendo a un ritmo sostenido, pero también es cierto que ese ritmo se ha desacelerado significativamente. La verdad es que no debe ilusionarnos demasiado estas estadísticas de crecimiento porque realmente ninguna economía es sostenible cuando alrededor del 15\% del PBI proviene de una actividad extractiva y no renovable como la minería, y, aproximadamente, el 17\% del narcotráfico.

Sobre el tema de la crisis de la economía de crecimiento, el filósofo griego Takis Fotopoulos realiza importantes aportes señalando que se trata de una crisis de la economía de mercado en su propia esencialidad definitoria y de la economía de crecimiento en tanto su consecuencia lógica. Bajo esta hipótesis, Fotopoulos (1997) propone el proyecto político de la democracia inclusiva asumiendo que "en el umbral de un nuevo milenio, la necesidad de reformular un nuevo proyecto liberador para la realidad de hoy es imperativa. El proyecto para una democracia inclusiva es, en consecuencia, propuesto no solo como otra utopía libertaria sino, en efecto como la única salida realista de la crisis crónica y, hoy, generalizada en un esfuerzo de integrar la sociedad con la política, la economía y la naturaleza”.

Para Fotopoulos, "la democracia inclusiva debe nutrirse desde sus mismas raíces, fecundarse como un espacio de igualdad y fundarse sobre

${ }^{2}$ La hoja de ruta se titula "Lineamientos centrales de política económica y social para un gobierno de concertación nacional"

(http://www.pcm.gob.pe/wp-content/uploads/2012/12/ollanta humala hoja de ruta01-a.pdf) 
una nueva cultura asamblearia: constituirse y completarse a sí misma como una democracia de las reuniones públicas, como una democracia autogestionaria y, en definitiva, también como una democracia directa y sin mediaciones. Todo ello, a su vez, abre paso a una noción de ciudadanía que lejos está de limitarse a ese ejercicio del voto a través del cual se renuncia a la asunción permanente de toda soberanía y se abdica de toda responsabilidad".

Spósito (1997), subraya que, para sustentar su proyecto político, Fotopoulos acuña el concepto de autonomía social, entendida esta como la capacidad colectiva de construir su propia utopía libertaria, pues, "si la autonomía es la base y la condición de posibilidad, y la libertad es el horizonte de transformación; la democracia inclusiva es la expresión más apropiada para delatar el carácter o el diagrama organizativo del proyecto liberador al que se adscribe..."

Sin lugar a dudas, para llevar a cabo el proyecto de la democracia inclusiva es importante rescatar la noción de ciudadanía, sin la cual, ningún proyecto político será pensado como posible. Y este tema tan complejo y tan abordado por los intelectuales de las ciencias políticas y sociales, merece puntualizarse desde la comunicación para el desarrollo a través de algunas reflexiones sin que estas pretendan abrir un debate que más bien le corresponde al terreno de los científicos sociales y de los politólogos.

Desde nuestro punto de vista particular, es imprescindible establecer un hilo conductor entre lo que entendemos por individuo, sujeto, ciudadano y ciudadanía. Desde nuestra perspectiva, la noción de individuo está relacionada a una unidad indivisible aplicada a un ser que es poseedor de un alma, un espíritu y una inteligencia que debe permitirle alcanzar su condición humana. El sujeto es, más bien, el ser humano que asume un rol de actor o agente en la sociedad y es depositario de una racionalidad y una cultura. El ciudadano es un sujeto que forma parte de una comunidad y de una cultura y es depositario y protagonista de derechos y obligaciones en la vida pública. La ciudadanía corresponde a una comunidad de ciudadanos que participa activamente en la esfera pública, en la "cosa pública", en busca del bien común, o sea, de aquello que le interesa y le pertenece a todos los ciudadanos. 
Como podemos observar, precisamos cuatro escalones bastante claros respecto a la necesidad de construir ciudadanía para la democracia y el desarrollo. Coincidimos en la necesidad de construir una ciudadanía activa y con sentido ético, capaz de generar las condiciones para crear el nuevo sentido social que nuestros países latinoamericanos aspiran alcanzar con madurez en este nuevo siglo o era civilizatoria que aún transita en su proceso fundacional. Por ello, más que hablar de la historia universal, deberíamos referirnos a la historia de las civilizaciones en la que se da cuenta de cómo las culturas luego de revertir sus estados de anomia ${ }^{3}$, alcanzan su máximo nivel de desarrollo una vez que han logrado establecer su sentido social, aquella metáfora que hace posible que los ciudadanos convivan armoniosa y civilizadamente y compartan los significados básicos que organizan la vida en sociedad.

Por otro lado, cuando abordamos la vinculación entre comunicación para el desarrollo y democracia, es imprescindible referirnos también a la noción de gobernabilidad ${ }^{4}$. El asunto de la gobernabilidad supera los problemas específicos de la acción del gobierno de turno y se proyecta ampliamente como una capacidad social y un atributo colectivo de toda la sociedad para dotarse de un sistema de gobierno. Hablamos entonces de la gobernabilidad de un país, región o de una ciudad, no de sus gobiernos, aunque, obviamente, las cualidades y calidades de estos son un factor importantísimo de la gobernabilidad.

En este entendido, un sistema social es gobernable cuando está estructurado sociopolíticamente de modo tal que todos los actores estratégicos se interrelacionan para tomar decisiones colectivas y resolver sus conflictos conforme a un sistema de normas, reglas y procedimientos formales o informales, que pueden registrar diversos niveles de institucionalización, dentro del cual formulan sus expectativas y estrategias.

${ }^{3}$ El concepto de anomia fue acuñado por Émile Durkheim para referirse a la falta de normas o a la incapacidad de la estructura social de proveer lo necesario para que los individuos alcancen sus metas en la sociedad. El término alude también a una desviación o ruptura de las normas sociales.

${ }^{4}$ Ver el Artículo "El aporte de la comunicación en la construcción de gobernabilidad". Lima, 2004. 
Si así definimos la gobernabilidad, ¿cuál es entonces el aporte de la comunicación para el desarrollo en la construcción de la gobernabilidad?

Hoy en día, es de consenso afirmar en la comunidad global que estamos viviendo un cambio de época o una auténtica revolución cultural y que transitamos de la sociedad industrial a la sociedad de la información, del conocimiento, post industrial, posmoderna, poscapitalista, etc.

Lo cierto es que, la historia de la humanidad da cuenta de una lucha incesante de los seres humanos por superar su condición primaria para afirmarse como seres superiores. Es una pugna permanente y sistemática entre civilización y barbarie, entre el ser primario y el ser superior. Así, el alcanzar al ser superior es el gran desafío y el común denominador de los seres humanos en todas las épocas.

La construcción del orden social apunta en su sentido más profundo a la construcción de humanidad. Pero dichos procesos serán posibles y viables en la medida en que los actores protagónicos de la historia sean capaces de dialogar y de entenderse para convivir en paz y armonía. Aludiendo a Rousseau, la construcción del orden social requiere de la existencia de un contrato social, de un pacto social, de un acuerdo entre seres humanos que, desde el punto de vista comunicacional, comparten un mismo sentido social.

En este marco de referencia, no cabe duda que entre comunicación y sociedad existe una relación indisoluble ya que sin comunicación es imposible la existencia de la sociedad y viceversa. Esto tiene que ver con el carácter ontológico de la comunicación, puesto que ésta es una cualidad propia e innata de los seres y grupos humanos en tanto están dotados de las competencias necesarias para poner en acción los procesos comunicativos. Pero asimismo, sin comunicación es imposible la organización de los individuos en sociedad ya que es en ella o a través de ella, -cual hilo invisible-, donde se tejen y se construyen las relaciones sociales.

De esta manera, la comunicación entendida básicamente como relaciones humanas directas o mediadas, se constituye en un espacio privilegiado para entender los procesos de construcción del tejido social y de los sistemas que los gobiernan. Hoy en día, la comunicación y los medios de comunicación se constituyen en las nuevas plazas públicas 
donde se construye lo político. En la perspectiva teórica de Robert Dhal y su apuesta por los regímenes poliárquicos, podríamos señalar que los procesos de democratización son más fuertes y significativos no solo cuando se da cabida al derecho de la participación política, sino también cuando se es permeable y se permite el debate público que, en este caso, atraviesa necesariamente por la esfera de los medios de comunicación masivos.

La comunicación aporta sustancialmente a la construcción del orden social y de la gobernabilidad, en tanto constituye un espacio privilegiado para que los actores estratégicos se acerquen y se reconozcan como interlocutores válidos (a pesar de sus diferencias), establezcan diálogos y confronten ideas y propuestas, y, lo que es aún más significativo, que como producto del diálogo logren ponerse de acuerdo y edifiquen los consensos necesarios estableciendo las normas y reglas de juego indispensables para la convivencia pacífica y civilizada. Todo ello nos remite a entender la comunicación para el desarrollo como un espacio privilegiado de concertación política de lo deseable y de lo posible que tiene en el diálogo, la tolerancia y la valoración de los otros, los ejes de la acción.

Por su parte, bajo los principios de la ética y la responsabilidad social, los comunicadores para el desarrollo, como conductores y operadores de los procesos de comunicación, cumplen también un rol fundamental en la construcción del orden social y la gobernabilidad.

Como líderes de opinión y mediadores sociales, los comunicadores para el desarrollo son los encargados de promover la vigilancia responsable contra la corrupción, el autoritarismo y la violación de los derechos humanos. Desde el enfoque de Schütz (1946), a los comunicadores les toca asumir la responsabilidad de promover la formación de ciudadanos bien informados, fortaleciendo sus capacidades para propiciar una participación responsable en la vida pública ya que con una información veraz, oportuna y de calidad, los ciudadanos podrán tomar decisiones conscientes y podrán cualificar y potenciar su participación en los asuntos públicos.

Los comunicadores para el desarrollo deben contribuir también a la transparencia democrática, posibilitando que la ciudadanía esté enterada del modo en cómo se administra y se gestiona el gobierno (central, regional y local) y en cómo se lidera el desarrollo, no solo exigiendo 
la rendición de cuentas relacionadas con el uso de los fondos públicos, sino también vigilando el cumplimiento de los programas de gobierno por el cual apoyaron a tal o cual candidato, en un ejercicio pleno de la democracia gobernante que, a diferencia de la democracia gobernada que se plasma de espaldas al pueblo como si se tratara de otorgar un cheque en blanco, se ejerce más bien mediante el diálogo y la consulta permanente, valorando y haciendo prevalecer la voluntad popular 5 .

Es también responsabilidad de los comunicadores para el desarrollo promover el debate público plural para contribuir a generar una cultura de la discusión, el debate y el diálogo, necesarios para construir los acuerdos y consensos necesarios que garanticen una convivencia pacífica y civilizada (Lechner, 1980).

Todos estos desafíos de los comunicadores para el desarrollo para contribuir responsablemente a la construcción del sistema social y la gobernabilidad pasa también por un asunto de carácter deontológico y por la práctica de la comunicación. Significa que los comunicadores deben manejar la información proponiendo una actitud preventiva y propositiva, -y no solo reactiva-, frente a los hechos; es decir, no esperar que sucedan los conflictos sociales, sino adelantarse a ellos. De esta manera, se podría advertir y hasta evitar eventos o problemas sociales que dañan la viabilidad democrática y la gobernabilidad.

Es necesario también que los comunicadores para el desarrollo establezcan líneas de continuidad y permanencia entre lo que acontece hoy y lo que ocurrió ayer. No es posible reducir el poder de la información a eso que muchos periodistas denominan como "la pepa"; es decir, a la dictadura del rating y de lo novedoso, de lo espectacular y de lo inmediato, sacrificando injustamente aquellos temas y hechos de coyuntura que por su importancia podrían alcanzar una trascendencia de mediano y largo plazo.

En la responsabilidad social de los comunicadores para el desarrollo, es fundamental también destacar su contribución en la formación de ciudadanos y ciudadanía, la recuperación de la autoestima y el

${ }_{5}^{5}$ Para mayor referencia sobre el tema, revisar el libro ¿Qué es la democracia? de Giovanni Sartori (2007), Editorial Taurus. 
reconocimiento de los individuos como sujetos y ciudadanos que pertenecen y comparten una misma comunidad.

\section{Referencias}

Armas, S. (2013). Red de Comunicadores por el Desarrollo de la Amazonía. Una experiencia estratégica de comunicación para el desarrollo. Lima, Perú: ILLA.

Armas, S. (1995). Imaginándonos el futuro. La comunicación como estrategia para el desarrollo. Lima, Perú: ILLA.

Armas, S. (2003). Apuntes para sustentar un enfoque de comunicación integral en los organismos públicos. Lima, Perú: Revista Comunifé $\mathrm{N}^{\circ} 3$.

Durkheim, E. (1998). El suicidio. Madrid, España: Akal.

Fotopoulos, T. (1997). Hacia una democracia inclusiva. La crisis de la economía de crecimiento y la necesidad de un nuevo proyecto liberador. Montevideo: Editorial Nordan-Comunidad.

Lechner, N. (1988). Los patios interiores de la democracia. Subjetividady politica. Santiago de Chile: FLACSO.

Martín, M. (1993). La comunicación en la vida cotidiana. La fenomenología de Alfred Schütz. Madrid, España: EUNSA.

Rogers, E. (1962). Difusión de las innovaciones. Nueva York: Presión libre. Roncagliolo, R. (1989). Desafíos de la investigación. Revista Chasqui $N^{\circ}$ 31. Quito, Ecuador: CIESPAL.

Schramm, Wilbur (1967). El papel de la información en el desarrollo nacional. Quito, Ecuador: CIESPAL.

Schütz, A. (1979). El problema de la realidad social. Buenos Aires, Argentina: Amorrortu.

Scott, J. (1986): A useful category of historical analysis. The American Historical Review Vol. 91, N 5.

Sen, A. (2000). Desarrollo y libertad. Barcelona, España: Editorial Planeta. UNESCO (1998). Informe de la Conferencia intergubernamental sobre politicas culturales para el desarrollo. Estocolmo, Suecia.

\section{Magister en Comunicaciones}

